\title{
112. A Simple Determination of Vitamin A in Margarine
}

\author{
By Kōji NaITo and Takajirō MORI \\ Laboratory of Fisheries Chemistry, Faculty of Agriculture, University of Tokyo \\ (Comm. by T. YABUTA, M.J.A., July 12, 1956)
}

In the assay procedure of vitamin $\mathrm{A}$ added to margarine, coloring materials have to be removed from unsaponifiable matters prior to colorimetric or spectrophotometric determination of vitamin A, as they interfer with the estimation. Chromatography has been widely adopted for this purpose, ${ }^{1-5)}$ but it requires skill and is not convenient for the routine work. The simple and rapid method suitable for practical use has been demanded.

The authors have succeeded in establishing a simple procedure to eradicate the artificial dyes, yellow $\mathrm{AB}$ and $\mathrm{OB}$, from margarine which does not contain carotenoids. This method is permitted to use in Japan officially.

It has been found that petroleum ether solution of yellow $\mathrm{AB}$ (phenylazo- $\beta$-naphthylamine) or $\mathrm{OB}$ (tolylazo- $\beta$-naphthylamine) gave a red or reddish violet color on addition of mineral acids and the changed dyes transfer themselves into acid layer in shaking. This extraction method of dyes, however, was not applicable because of marked decomposition of vitamin A by mineral acid. After several trials, a suitable solvent was found fortunately, which was able to alter the pigments to reddish substances and to take up it quantitatively from petroleum ether layer without any loss of the vitamin. The solvent was Japanese commercial glacial acetic acid.

The change of pigments was shown successfully to be not due to glacial acetic acid itself, but to some contamination, by the fact that the glacial acetic acid purified with chromic acid failed to act on the dyes. This accompanying substance which plays an important role in the process was proved to be acetaldehyde present in a very small quantity.

From the above observations, the authors have devised a simple method to remove the pigments by solvent extraction.

1. Standard procedure

From 3 to $5 \mathrm{~g}$ margarine was saponified with alcoholic potassium hydroxide in the usual manner described thoroughly in U. S. P. XIV ${ }^{6}{ }^{6}$ The unsaponifiable matters were dissolved in about $30 \mathrm{ml}$ petroleum ether (b.p. $35 \sim 60^{\circ}$ ) and transferred into $100 \mathrm{ml}$ separatory funnel.

To the petroleum ether solution, $10 \mathrm{ml}$ of distilled glacial acetic acid (b.p. $116 \sim 117^{\circ}$ ) and $1 \mathrm{ml}$ of $1 \%$ acetaldehyde solution were added and the mixture was swirled gently. In this treatment, pigments 
transferred to acetic acid layer, assuming the reddish violet coloration. Then, $10 \mathrm{ml}$ of $10 \sim 15 \%$ sodium chloride solution was poured into the funnel in order to avoid the loss of vitamin A. After shaking, the lower layer was drawn off. When the complete removal of pigments was not attained because of insufficient shaking or low concentration of acetaldehyde, this treatment was repeated once more. The upper layer was washed by gentle shaking from 4 to 5 times with $30 \mathrm{ml}$ portions of water, until the washing became free from acid. After removal of the final wash water, the petroleum ether extracts were transferred into a flask and desiccated by adding several grams of anhydrous sodium sulfate and allowing the mixture to stand for a few minutes. Petroleum ether was then driven off under the diminished pressure with introduction of the current of an inert gas and the residual unsaponifiable matters were dissolved into $10 \mathrm{ml}$ purified chloroform. If necessary, the remaining pigments were detectable by the coloration of acid layer, when $2 \sim 3 \mathrm{ml}$ of chloroform solution was shaken vigorously with $1 \mathrm{ml}$ concentrated hydrochloric acid.

Finally, vitamin A was determined by the colorimetric method using antimony trichloride reagent or activated glyceroldichlorohydrin reagent, as usual.

2. The reaction between pigments and aldehyde

In their chemical structures, yellow $\mathrm{AB}$ and $\mathrm{OB}$ posses common $\beta$-naphthylamie radical as described below and differ from each other in that the former is phenylazo-compound, whereas the latter is 0 tolylazo-compound. As both the dyes behaved to the aldehyde in a simmilar manner, the reaction was assumed to occur in the common part of both dyes, namely in $\beta$-naphthylamine radical. Furthermore, the reaction was supposed to be the condensation of the amine and aldehyde at amino group, yielding phenylazo-aldehyde- $\beta$-naphthylimid (I) or $o$-tolylazo-aldehyde- $\beta$-naphthylimid (II), as follows.

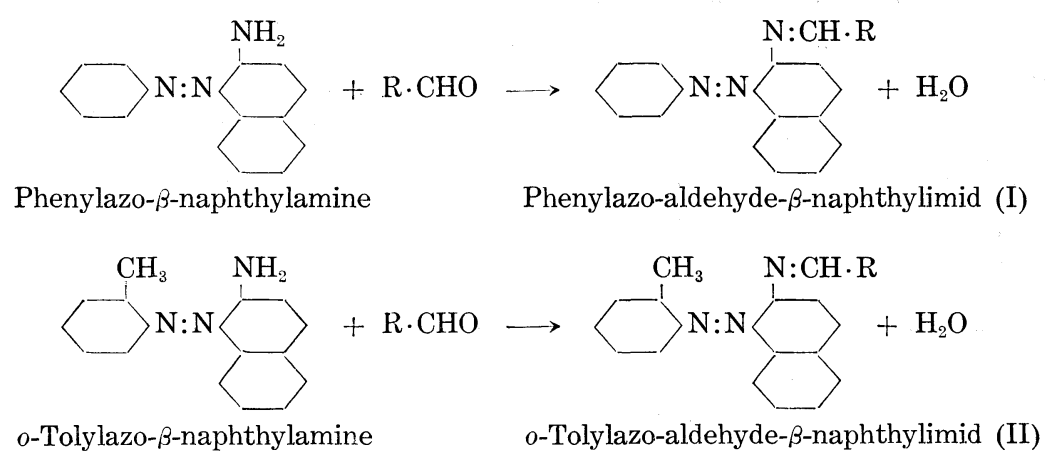

The compounds (I) and (II) have never been described so far in the literature. 
a) The syntheses and identification of phenylazo-acetaldehyde- $\beta$ naphthylimid

An adequate quantity of acetaldehyde $(10 \mathrm{ml})$ was added to an alcoholic solution of phenylazo- $\beta$-naphthylamine (5 g) and boiled for 20 30 minutes. A red compound appeared in a viscous solution when the reaction mixture was left standing over night. The precipitate (5 g) was separated from viscous mother liquor by filtration and recrystallized from hot alcohol. The crystal was treated with active carbon in alcohol. Finally, colorless crystals of hexagonal plate were obtained, m.p. $236^{\circ}$ (uncor.), $\lambda_{\max } 244 \sim 246 \mathrm{~m} \mu$ (in alcohol) and $\mathrm{E}_{1 \mathrm{~cm}}^{1 \% \%} 245$ $\mathrm{m} \mu, 2.032$.

Phenylazo-acetaldehyde- $\beta$-naphthylimid $\quad \mathrm{C}_{18} \mathrm{H}_{15} \mathrm{~N}_{3}$

$\begin{array}{llll}\mathrm{C} & \mathrm{H} & \mathrm{N} & \text { Mol. wt. }\end{array}$

$\begin{array}{lllll}\text { Caled. } & 79.12 & 5.49 & 15.38 & 271.3 \\ \text { Found } & 78.23 & 5.41 & 14.39 & 286\end{array}$

The infrared spectra of this compound and phenylazo- $\beta$-naphthylamine are shown in the following figure. The spectrum of phenylazo$\beta$-naphthylamine revealed the absorptions at 2.90 and $6.14 \mu$, indicating
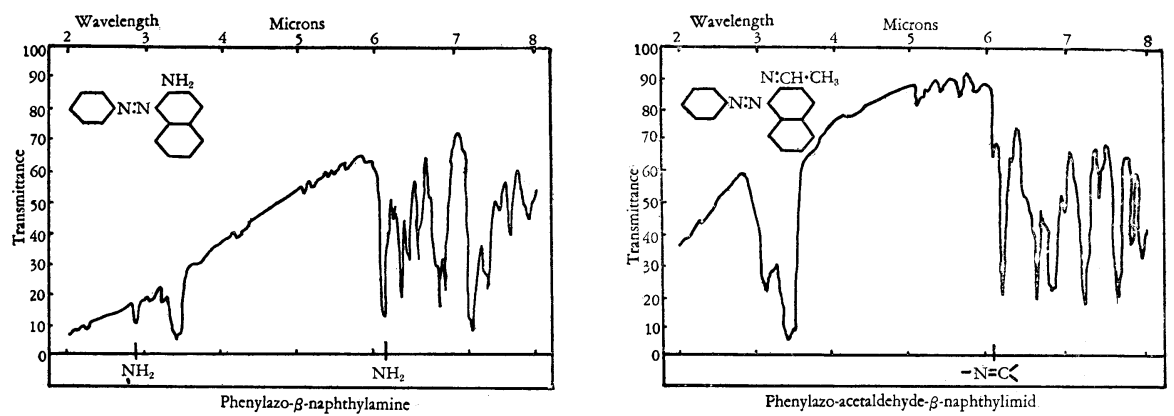

Infrared spectra of phenylazo- $\beta$-naphthylamine and phenylazo-acetaldehyde$\beta$-naphthylimid

the presence of $\mathrm{NH}_{2}$ radical, but these absorptions disappeared from the spectrum of this compound and a characteristic absorption of $-\mathrm{N}=\mathrm{C}$ linkage at $6.10 \mu$, was clearly observed. This is a powerful evidence for the formation of phenylazo-acetaldehyde- $\beta$-naphthylimid (III).

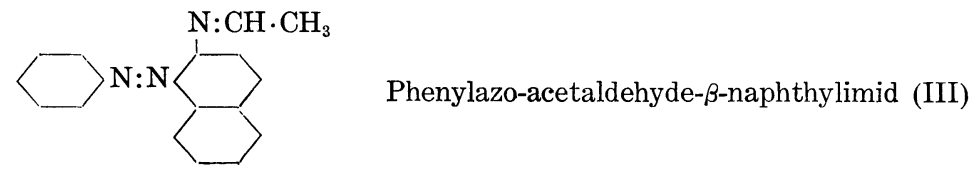

The crystal is soluble in chloroform, tetrachlorcarbon, carbon disulfide, acetone, glacial acetic acid, benzol, and alcohol, and insoluble in petroleum ether, benzin, ligroin, and water. This solubility well coincides with the principle adopted in the standard procedure, 
b) $o$-Tolylazo-acetaldehyde- $\beta$-naphthylimid

This compound was prepared from $o$-tolylazo- $\beta$-naphthylamine and acetaldehyde in the same manner as described above. It crystallizes in colorless hexagonal plate from alcohol, m.p. $176.5^{\circ}$ (uncor.), $\lambda_{\max }$ 245 246 $\mathrm{m} \mu$ (in alcohol) and $\mathrm{E}_{1 \mathrm{~cm},}^{1 \%} 245 \mathrm{~m} \mu, 1.920$.

$o$-Tolylazo-acetaldehyde- $\beta$-naphthylimid $\mathrm{C}_{19} \mathrm{H}_{17} \mathrm{~N}_{3}$

$\begin{array}{lcccc} & \mathrm{C} & \mathrm{H} & \mathrm{N} & \text { Mol. wt. } \\ \text { Calcd. } & 79.44 & 5.92 & 14.63 & 287.5 \\ \text { Found } & 79.43 & 6.31 & 13.83 & 280.7\end{array}$

The solubility of this crystal is just the same as that of phenylazoacetaldehyde- $\beta$-naphthylimid.

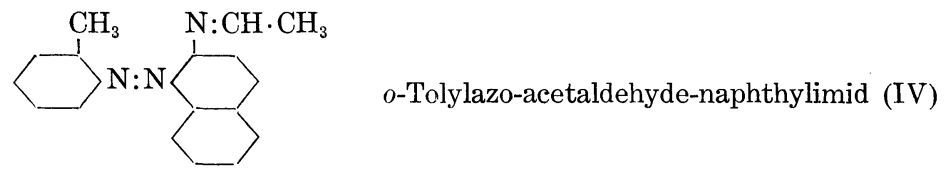

c) The yield of phenylazo-acetaldelyde- $\beta$-naphthylimid in the standard procedure

In the standard procedure, the pigments were observed to become reddish violet substances in acetic acid on addition of acetaldehyde, but (III) and (IV) were obtained in colorless crystals. Therefore, the yield of (III) in the standard procedure was determined spectrophotometrically and (III) was found to be the principal product.

After the pigment (yellow AB) was transferred to acetic acid layer according to the standard procedure, the acid layer was neutralized with 10\% sodium hydroxide solution. The red viscous substance separated from the solution, was extracted with ether and the ether fraction was washed with water several times. After the removal of ether, the residue was dissolved in alcohol and measured its absorbency at $245 \mathrm{~m} \mu$. The yield of (III) corresponded to $78 \%$ of the theoretical value. It was proved that about $80 \%$ of phenylazo- $\beta$-naphthylamine condensed with acetaldehyde to (III) in this procedure and the substance assuming reddish violet color, which seemed to be dominant in appearance, was very small in quantity and should be considred to be the by-product accompanying the reaction.

\section{Summary}

The authors have devised a simple method to remove the artificial dyes, yellow $\mathrm{AB}$ and $\mathrm{OB}$ from the unsaponifiable matter of margarine by treating the petroleum ether solution with glacial acetic acid containing a small amount of acetaldehyde. Applying this method, the vitamin $\mathrm{A}$ in margarine was determined easily and accurately. In this determination, yellow $\mathrm{AB}$ and $\mathrm{OB}$ were found to become phenylazo- 
acetaldehyde- $\beta$-naphthylimid or $o$-tolylazo-acetaldehyde- $\beta$-naphthylimid by condensing with acetaldehyde and can be removed easily by acetic acid.

\section{References}

1) J. B. Wilkie: J. Assoc. Offic. Agr. Chemists, 32, 455 (1949).

2) J. Boldingh and J. R. Drost: J. Am. Oil Chem. Soc., 28, 480 (1951).

3) L. Rosner and H. Kan: J. Assoc. Offic. Agr. Chemists, 37, 887 (1954).

4) J. W. Lord and P. M. Bradely: Analyst, 80, 429 (1955).

5) A. Fujita and M. Aoyama: Vitamins (in Japanese), 6, 139 (1953).

6) Pharmacopeia of the United States XIV, 784 (1950). 